\title{
Eco-friendly method to synthesize and characterize 2D nanostructured (1,2-bis(diphenyl-phosphino)ethyl) tungsten tetracarbonyl methyl red/copper oxide di-layer thin films
}

\author{
AHMED FAROUK AL-HOSSAINY ${ }^{1,2, *}$, MOHAMED SHAFICK ZOROMBA ${ }^{3,4}$ \\ and REDA HASSANIEN ${ }^{1}$ \\ ${ }^{1}$ Department of Chemistry, Faculty of Science, Assiut University, New Valley Branch, El-Kharja 72511, Egypt \\ ${ }^{2}$ Chemistry Department, Faculty of Science - Arar, Northern Border University, Arar 1321, Saudi Arabia \\ ${ }^{3}$ Chemical and Materials Engineering Department, King Abdulaziz University, Rabigh 21911, Saudi Arabia \\ ${ }^{4}$ Chemistry Department, Faculty of Science, Port-Said University, Port-Said 42521, Egypt \\ *Author for correspondence (ahmed73chem@scinv.au.edu.eg)
}

MS received 16 July 2017; accepted 12 October 2017; published online 23 May 2018

\begin{abstract}
Three-layer thicknesses $\left(T_{1}=50, T_{2}=75\right.$ and $\left.T_{3}=100 \mathrm{~nm}\right)$ of 1,2-bis(diphenylphosphino)ethyl tungsten tetracarbonyl methyl red (DPE-W-MR) were deposited onto the $\mathrm{CuO}$ thin film $(50 \mathrm{~nm})$ to produce $\mathrm{DPE}-\mathrm{W}-\mathrm{MR} / \mathrm{CuO}$ di-layer thin films by sol-gel spin-coating technique. The composition and the chemical structure of the as-prepared thin films were characterized using various techniques including elemental analysis, Fourier transform infrared spectroscopy, ${ }^{1} \mathrm{H}-\mathrm{NMR}$ and X-ray diffraction (XRD). Scanning electron microscopy was used to investigate the size and shape of the CuO nanoparticles and the fabricated thin films. The films are crystalline as evidenced by the XRD pattern and DPE-W-MR has an orthorhombic crystal system. The crystallite size was calculated from an analysis of the line broadening features using the Scherrer formula; the average crystallite sizes of DPE-W-MR/CuO di-layer thin films are 52.92, 56.24 and $72.26 \mathrm{~nm}$ for $T_{1}$, $T_{2}$ and $T_{3}$, respectively. Thermogravimetric analysis and the thermal curve of DPE-W-MR complex were studied. Optical properties of DPE-W-MR/CuO di-layer thin films are discussed. The optical band gap energies of DPE-W-MR di-layer thin films $/ \mathrm{CuO}$ decreased $\left(2.25,2.1\right.$ and $1.88 \mathrm{eV}$ ) as the film thickness increased (from $T_{1}$ to $T_{3}$ ). Based on the optical results and the quantum confinement effects, the DPE-W-MR/CuO di-layer thin films may be candidates as semiconductor materials for optoelectronic devices.
\end{abstract}

Keywords. Phosphine; copper oxide; thin film; XRD; SEM; optical properties.

\section{Introduction}

Two-dimensional (2D) organic-inorganic hybrid structures have attracted attention due to their interesting structural and optical properties [1-5]. Copper is one of the most technologically important metals to form as 1D nanowires at the nanoscale [6] and/or 2D thin films [7]. Copper element remains the material of choice for consumer electronics due to its favourable conductivity/cost ratio. Metallic copper thin films have various applications, especially in electrical contacts in printed circuit boards [8,9]. Based on the high conductivity of copper films, they were utilized as highly uniform and reproducible substrates for plasmon-enhanced fluorescence [10]. In addition, the copper thin films are used in photocatalysis and solar photovoltaics technologies. Nanocrystalline semiconductor particles have drawn considerable interest because of their useful properties, such as electronic properties and unique optical properties, as compared to those of the bulk materials. The oxides of transition metals are an important class of semiconductors, which have applications in magnetic storage media, solar energy transformation, electronics and catalysis. Therefore, preparation of metallic/semiconductor thin films with fine-tuned properties for specific applications is considered a challenge for chemists. There are different preparations, which were considered, including wet and dry methods were used for the deposition of metal/semiconductor thin films. These methods, such as atomic layer deposition (ALD), photochemical deposition [11], physical vapour deposition (PVD) [12], in addition to molecular precursor [13], ion beam-assisted deposition (IBAD) [14] and hydrothermal methods [15] were applied. When the same thin film is deposited by different techniques, these techniques give the film with different phase structures, degree of crystallinity and variable optoelectronic properties.

At the same time, much great progress was devoted to the applications of phosphine compounds in various catalysed reactions because these compounds are easy to prepare and modify. Particularly, triphenyl phosphine is the most commonly used as it was prepared with high yields and high 
selectivity in a wide range of catalysed reactions. Recently, nano-sized diphosphine complexes containing aromatic substituents were attracted much attention [16]. Diphosphine complexes have low oxidation states with transition element and could be used to fine-tune electronic and their steric properties in corresponding coordinated compounds. The connected substituents with phosphorus atom can alter electron donating or electron withdrawing property of the ligand. Bulky phosphines can be used as effective compounds with specific transition metal based on the reaction type between the phosphine compound and transition metal [17]. Phosphine ligands with bulky aromatic substituents showed a good catalytic property in the alcohol oxidation and hydrosilylation reactions $[18,19]$ with a proper transition metal. Complexes with metal hexacarbonyl (group VI) with diphenyl phosphine ligands were used to synthesize phosphide-metals that were used to synthesize nanocrystalline spintronic materials [20]. Complexes of tetra-carbonyl metals were reported as therapeutic CO-releasing molecules $[21,22]$.

Herein, the preparation and characterization of DPE-W$\mathrm{MR} / \mathrm{CuO}$ nanocomposite di-thin films were fabricated based on the facial synthetic route to produce $2 \mathrm{D}$ thin films by sol-gel spin-coating technique. The composition and the chemical structure of the fabricated di-thin films were characterized using elemental analysis (EA), Fourier transform infrared spectroscopy (FTIR), ${ }^{1} \mathrm{H}-\mathrm{NMR}$, thermogravimetric analysis (TGA) techniques, X-ray powder diffraction (XRD) and optical parameters. The surface morphology of the dithin film was investigated using scanning electron microscope (SEM).

\section{Experimental}

\subsection{Materials}

1,2-bis(diphenylphosphino)ethyl (DPE), methyl red (MR), ethanol, 2-methoxethanol, monoethanol amine and copper(II) acetate monohydrate $\left(\mathrm{Cu}\left(\mathrm{CH}_{3} \mathrm{COO}\right)_{2} \cdot \mathrm{H}_{2} \mathrm{O}\right)$ were purchased from Sigma-Aldrich and used as received without further purification.

\subsection{Methods}

2.2a Preparation of (DPE-W-MR) $3 \mathrm{H}_{2} \mathrm{O}$ complex: 1,2-bis (diphenylphosphino)ethyl tungsten tetracarbonyl methyl red (DPE-W-MR) complex was prepared by dissolving MR in ethanol (0.269 mmol in $5 \mathrm{ml}$; w/v). Subsequently, MR solution was added dropwise to DPE-W solution (0.692 mmol in $6 \mathrm{ml}$; w/v dichloromethane) under magnetic stirrer (500 rpm) at room temperature [23-26]. The resulting mixture was completely stirred for $\sim 15 \mathrm{~min}$ at $110^{\circ} \mathrm{C}$, then, the volume of the solution was concentrated to $\cong 2 \mathrm{ml}$ under reduced pressure. A red precipitate DPE-W-MR complex was washed several times by appropriate volume of n-hexane and dried under vacuum. Yield: $86 \%$ of a red powder. Chemical formula
(CF): $\mathrm{C}_{43} \mathrm{H}_{42} \mathrm{~N}_{3} \mathrm{O}_{7} \mathrm{P}_{2} \mathrm{~W}$, molecular weight: 958.61, EA: C, 53.88 (53.83); H, 4.42 (4.28); N, 4.38 (4.30).

\subsection{Fabrication of DPE-W-MR/CuO di-layer thin films}

DPE-W-MR/copper oxide (DPE-W-MR/CuO) di-layer thin films were fabricated by sol-gel spin-coating technique using TC100 spin coater on quartz substrates. $\mathrm{Cu}\left(\mathrm{CH}_{3} \mathrm{COO}\right)_{2}$. $2 \mathrm{H}_{2} \mathrm{O}$ used as a precursor to produce $\mathrm{CuO}$, while 2-methoxethanol used as a solvent. Monoethanol amine was used as stabilizer during the fabrication process. The ratio between the stabilizer (mono-ethanol amine) and precursor/s either $\mathrm{Cu}\left(\mathrm{CH}_{3} \mathrm{COO}\right)_{2} \cdot 2 \mathrm{H}_{2} \mathrm{O}$ or DPE-W-MR was $1: 1$. The quartz substrate was cleaned in methanol and acetone baths for $10 \mathrm{~min}$ by an ultrasonic cleaner, then the substrate was rinsed with deionized water and dried. Subsequently, $\mathrm{Cu}\left(\mathrm{CH}_{3} \mathrm{COO}\right)_{2} \cdot \mathrm{H}_{2} \mathrm{O}$ precursor was dropped onto the quartz substrate at $1500 \mathrm{rpm}$ per $150 \mathrm{~s}$. After the spin coating, the film was dried at $150^{\circ} \mathrm{C}$ for $10 \mathrm{~min}$ on a hot plate to evaporate the solvent and remove the organic residuals. The resulting $\mathrm{CuO}$ film was calcined at $500^{\circ} \mathrm{C}$ for $2 \mathrm{~h}$ in air furnace and left to cool to room temperature kept in an evacuated desiccator for testing to avoid the moisture effect. The thickness of the resulting film was found to be $50 \mathrm{~nm}$, which was measured by a digital micrometer with an accuracy of $\pm 0.001 \mathrm{~mm}$. To fabricate DPE-W-MR/CuO di-layer thin films, DPE-W-MR solution was deposited onto the resulting $\mathrm{CuO}(50 \mathrm{~nm})$ film with 50, 75 and $100 \mathrm{~nm}$. The deposition was applied according to the previous step under the same previous conditions. These three different thicknesses were labelled as $T_{1}, T_{2}$ and $\mathrm{T}_{3}$, respectively.

\subsection{Characterization}

EA of the fabricated thin films were performed with 2400 Perkin-Elmer, USA elemental analyzer. Perkin-Elmer FTIR type 1650 spectrophotometer was used to FTIR analysis of the wavenumber range of $4000-400 \mathrm{~cm}^{-1}$. ${ }^{1} \mathrm{H}-\mathrm{NMR}$ spectra were recorded by a Bruker (400 MHZ) spectrometer. TMS was used as an internal standard reference. Dimethyl sulfoxide-d ${ }^{6}\left(\mathrm{CD}_{3}\right)_{2} \mathrm{SO}_{4}$ was used as a deuterated solvent and the spectra extended from 0 to $14 \mathrm{ppm}$. TGA of DPE-W$\mathrm{MR}$ complex was studied in the range of $45-1000^{\circ} \mathrm{C}$ under nitrogen atmosphere $\left(20 \mathrm{ml} \mathrm{min}{ }^{-1}\right)$ with a heating rate of $20^{\circ} \mathrm{C} \mathrm{min}^{-1}$ by DTG-60H Shimadzu simultaneous TG/DTG apparatus. XRD of DPE-W-MR/CuO di-layer thin films was studied by Philips X'Pert Pro diffractometer equipped with $\mathrm{CuK} \alpha$ radiations. Optical parameters of the DPE-W-MR/CuO di-layer thin films were calculated at room temperature by a Shimadzu UV-3101UV-vis-NIR pc spectrophotometer in the wavelength range of 250-2500 $\mathrm{nm}$. The morphology of the DPE-W-MR/CuO di-layer thin films was investigated using SEM by JEOL-JSM-5400 LV electron microscope. 


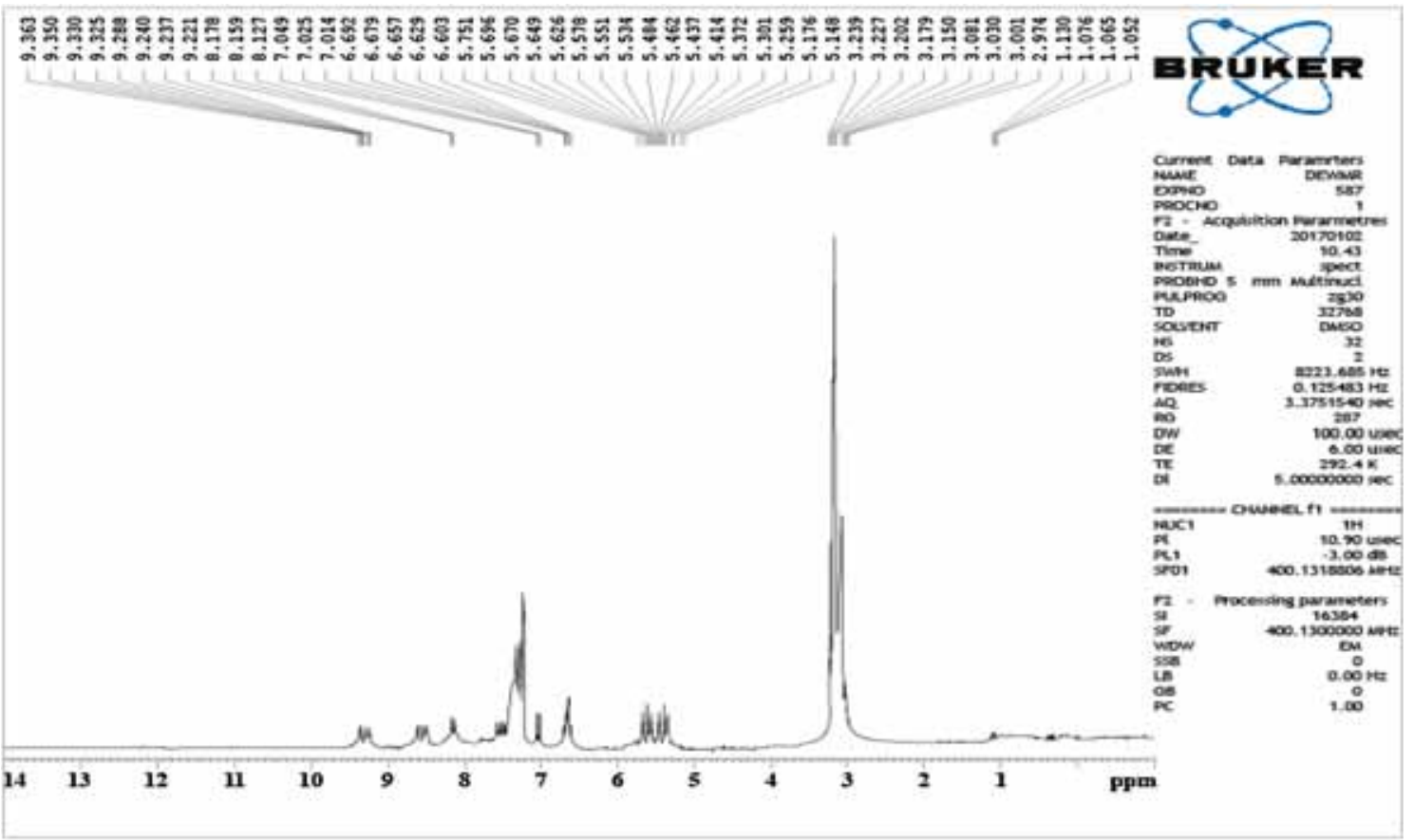

Figure 1. ${ }^{1} \mathrm{H}-\mathrm{NMR}$ spectrum of the synthesized DPE-W-MR complex.

\section{Results and discussion}

\subsection{FTIR of (DPE-W-MR) $3 \mathrm{H}_{2} \mathrm{O}$ complex}

IR $\left(\mathrm{KBr}, \mathrm{cm}^{-1}\right)$ : the band around $3625 \mathrm{~cm}^{-1}$ can be attributed to the molecules as a moisture in the complex during the synthesis process [27,28]. The medium intensity band observed at $3143 \mathrm{~cm}^{-1}$ is attributable to $v(\mathrm{C}-\mathrm{H})_{\text {aromatic vibration. The }}$ weak intensity band appeared at $2985 \mathrm{~cm}^{-1}$ is referred to $v(\mathrm{C}-\mathrm{H})_{\text {aliphatic }}$ vibrational mode. The strong intensity band at $1750 \mathrm{~cm}^{-1}$ is due to the overlapping of $v(\mathrm{C}=\mathrm{O})$ of both carboxylic acid and ester. The band medium intensity at 1555 $\mathrm{cm}^{-1}$ is referred to $v(\mathrm{C}=\mathrm{C})$ mode. Finally, the bands located at (strong 2045; medium 1990; medium 1985) and (weak 1970; weak 725 ; strong $\left.680 \mathrm{~cm}^{-1}\right)$ may be mainly due to $v_{\text {sym }}(\mathrm{C} \equiv \mathrm{O})$ and $v(\mathrm{P}-\mathrm{C})$, respectively.

\section{$3.2{ }^{1} H-N M R$ of the DPE-W-MR complex}

Figure 1 shows ${ }^{1} \mathrm{H}-\mathrm{NMR}$ spectrum of the DPE-W-MR complex. The spectrum shows multiple types of resonances at $\delta \cong$ 3.11 and $7.29 \mathrm{ppm}$ for six protons in dimethyl groups and 16 protons in four phenyl groups of the complex, respectively. The two strongest peaks located at $\delta \cong 2.45$ and $3.4 \mathrm{ppm}$ can be attributed to DMSO protons and water, respectively, in DMSO. A doublet of doublet singles of relative intensity $2 \mathrm{H}$ at a range $\delta \cong 5.45 \mathrm{ppm}$ were observed and assigned to $(-\mathrm{CH}=\mathrm{CH}-)$ protons coupling together and in the presence of two phosphorous atoms, ${ }^{2} \mathrm{~J}(\mathrm{CH}=\mathrm{CH}) 46 \mathrm{~Hz}$. The two weak peaks located at $\delta \cong 6.65$ and 7.03 ppm can be attributed to the two protons in benzene ring containing $\left(\mathrm{CH}_{3}\right)_{2}-\mathrm{N}-$ group. The weak peaks observed at $\delta \cong 7.52,8.15,8.55$ and $9.29 \mathrm{ppm}$ have a relative low intensity $\mathrm{CH}$ proton in benzene ring containing a carboxylic group.

\subsection{TGA of DPE-W-MR complex}

Thermal decomposition profile of DPE-W-MR complex was studied by the TGA in the range of $45-1000^{\circ} \mathrm{C}$. TG/DTG data of the complex [(DPE-W-MR $\left.)\left(\mathrm{H}_{2} \mathrm{O}\right)_{2}\right] \mathrm{H}_{2} \mathrm{O}$ is tabulated in table 1. The decomposition of [(DPE-W-MR $\left.)\left(\mathrm{H}_{2} \mathrm{O}\right)_{2}\right] \mathrm{H}_{2} \mathrm{O}$ complex was carried out through four stages (figure 2). The first one, within the temperature range of $45-104.4^{\circ} \mathrm{C}$ (DTG peak at $100^{\circ} \mathrm{C}$ ), is related to weight loss (obs.: $1.85 \%$; calc.: $1.87 \%$ ). This weight loss can be attributed to the removal of lattice water molecules. The second one, within the temperature range of $140-193^{\circ} \mathrm{C}$, is related to the removal of two coordinated water molecules and one mole of carbon monoxide [29,32]. This stage of weight loss was found to be $6.67 \%$ (theoretical value of $6.68 \%$ ). The third stage of weight loss was found to be $27.78 \%$ within the temperature range of 255 $383^{\circ} \mathrm{C}$ (with a DTG peak at $383.89^{\circ} \mathrm{C}$ ). This can be attributed to the removal of MR part. The fourth one, within the temperature range of $424-470^{\circ} \mathrm{C}$, is related to the removal of DPE ligand. The weight loss within the fourth stage was found to be $22.52 \%$. The total weight loss through the four steps by TGA was found to be $77.45 \%$. Thus, these results are in a good agreement with the theoretical value of $77.48 \%$. The remaining weight found to be $22.52 \%$ is related to tungsten dioxide. 
Table 1. Thermoanalytical results (TG of the tungsten metal complexes of DPE-W-MR).

\begin{tabular}{|c|c|c|c|c|}
\hline Complex & TG & $\begin{array}{l}\text { Mass loss }(\mathrm{mg}) \\
\quad(13.178)^{a}\end{array}$ & $\begin{array}{l}\text { Mass loss\% } \\
\text { obs. (calc.) }\end{array}$ & Assignments \\
\hline \multirow{6}{*}{$\begin{array}{l}{[\mathrm{DPE}-\mathrm{W}-\mathrm{MR}} \\
\left.\left(\mathrm{H}_{2} \mathrm{O}\right)_{2}\right] \mathrm{H}_{2} \mathrm{O}\end{array}$} & $45-104.4$ & 0.2474 & $1.85(1.87)$ & Loss of one lattice water molecule \\
\hline & $140-193$ & 0.8805 & $6.67(6.68)$ & Loss of two coordinated water molecules+ one mole $\mathrm{CO}$ \\
\hline & $255-383$ & 3.6608 & 27.78 & Removal of one part of the MR ligand \\
\hline & $424-470$ & 5.4223 & 41.15 & Removal of part of the DPE ligand \\
\hline & $506-837$ & 2.9672 & 22.52 & leaving $\mathrm{WO}_{2}$ residue \\
\hline & & $10.211^{b}$ & $77.45^{c}(77.48)$ & \\
\hline
\end{tabular}

${ }^{a}$ Weight of the sample in thermal detector.

${ }^{b}$ Total weight of decomposition leaving metal oxide.

${ }^{c}$ Total mass loss $\%$.

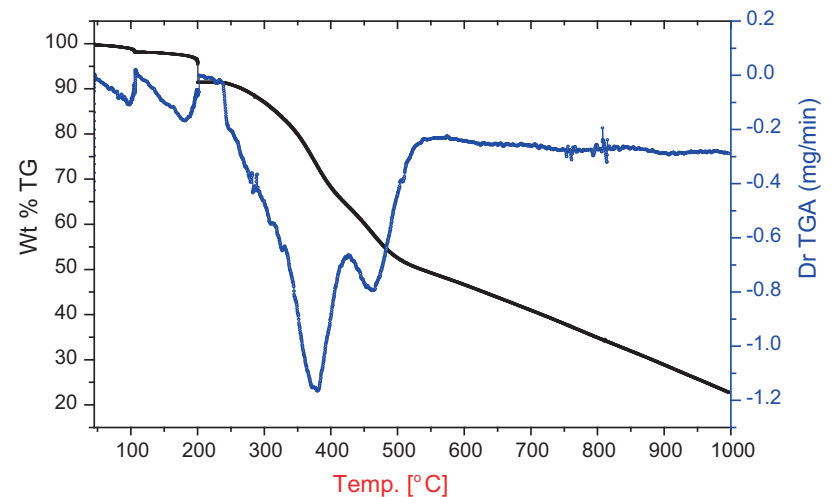

Figure 2. Thermogravimetric analysis (TGA) and DrTGA thermal curve DPE-W-MR complex. The decomposition of [(DPE-W-MR) $\left.\left(\mathrm{H}_{2} \mathrm{O}\right)_{2}\right] \mathrm{H}_{2} \mathrm{O}$ complex carried out through four stages.

\subsection{XRD of the DPE-W-MR complex and its composites thin films with $\mathrm{CuO}$}

Figure 3 shows the XRD diffraction pattern of DPE-W-MR powder. It is observed that the pattern displays many diffraction peaks with different intensities. This indicates that the DPE-W-MR powder has a polycrystalline nature. Indexing of each diffraction peak was achieved by CHECKCELL program. The program confirms that the DPE-W-MR has an orthorhombic crystal system and space group $\mathrm{Aba}_{2}$ with lattice parameters: $\alpha=\gamma=\beta=90.00^{\circ}, a \neq b \neq c, 15.150$, 13.565 and $13.375 \AA$, respectively. Based on figure 3 data, it can be noticed that the complex has a crystalline nature with preferred orientations of $(0 \overline{2} 2),(\overline{2} 02),(2 \overline{1} 3),(121),(\overline{1} 00)$, $(\overline{3} 02),(41 \overline{2}),(\overline{3} 22),(2 \overline{5} 2),(\overline{1} \overline{4} 2)$ and $(0 \overline{3} \overline{4})$ at $2 \theta$ and intensity equals to $\left(7.72^{\circ}, 818\right),\left(16.48^{\circ}, 509\right),\left(19.66^{\circ}, 1076\right),\left(20.32^{\circ}\right.$, $745),\left(22.42^{\circ}, 140\right),\left(24.04^{\circ}, 469\right),\left(24.64^{\circ}, 1410\right),\left(27.46^{\circ}\right.$, $1022),\left(29.2^{\circ}, 538\right),\left(31.6^{\circ}, 766\right)$ and $\left(33.88^{\circ}, 114\right)$, respectively.

The crystalline nature of the prepared copper oxide $(\mathrm{CuO})$ nanoparticles (modified sol-gel) was identified from their corresponding powder XRD patterns (figure 4). The sharp

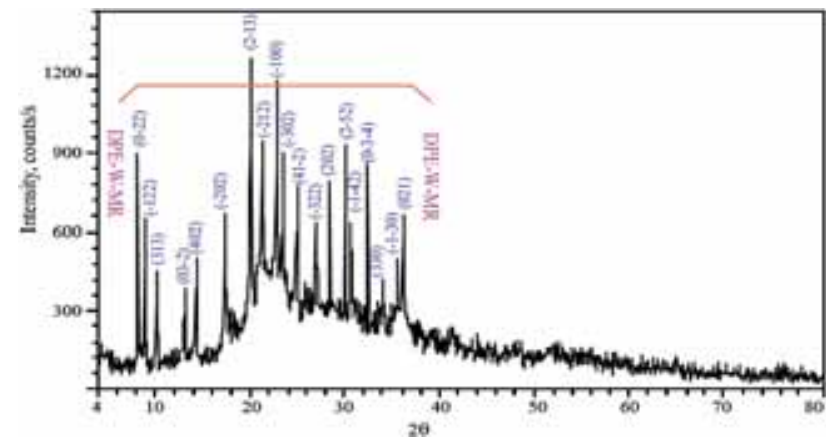

Figure 3. XRD patterns of the DPE-W-MR powder.

peak positions indicate the good crystallinity of the prepared $\mathrm{CuO}$ nanoparticles. Obviously, the diffraction peaks can be identified for monoclinic (tenorite, syn) phase of copper oxide (JCPDS file no. 04-015-5877) with lattice parameters: $a=4.6881, b=3.423, c=5.1323 \AA, \beta=99.459^{\circ}$ and volume $=81.24 \AA^{3}$. The crystallite size $(D)$ and $d$-spacing of $\mathrm{CuO}$ nanoparticles were calculated using Debye-Scherrer's formula; $D=0.9 \lambda /(\beta \cos \theta)$ and Bragg equation: $n \lambda=2 d \sin$ $\theta$, respectively. The $d$-spacing and $\beta$ are the full-width at halfmaximum intensity and the calculated $D$ values, respectively, and are listed in table 2. Finally, the average particle size of copper oxide thin film $\left(D_{\text {av }}\right)$ is found to be $\cong 47.68 \mathrm{~nm}$.

On the other hand, figure 5 shows the XRD pattern of the as-deposited di-layers of DPE-W-MR/CuO thin films $\left(\mathrm{T}_{1}, \mathrm{~T}_{2}\right.$ and $\mathrm{T}_{3}$ ). The height of intensity of the $\mathrm{CuO}$ thin films appears to depend on synergistic effects and layer thickness. Within the diffraction angle range of $2 \theta=7.5-35$ degree, the intensity of the peaks gradually increases with the increase in the DPE layer thickness. The intensity of the peaks of the thin films depends on synergistic effects between the adjacent layers during the deposition process and overall stress levels. In other words, figure 5 clearly shows that as the thickness of the deposited DPE-W-MR film increases from $\mathrm{T}_{1}$ to $\mathrm{T}_{3}$, within $2 \theta=35.36-80^{\circ}$, the peaks $(002),(111),(\overline{2} 02),(020)$, (202), (113), (022), (113), (311) and (004) gradually decrease. 


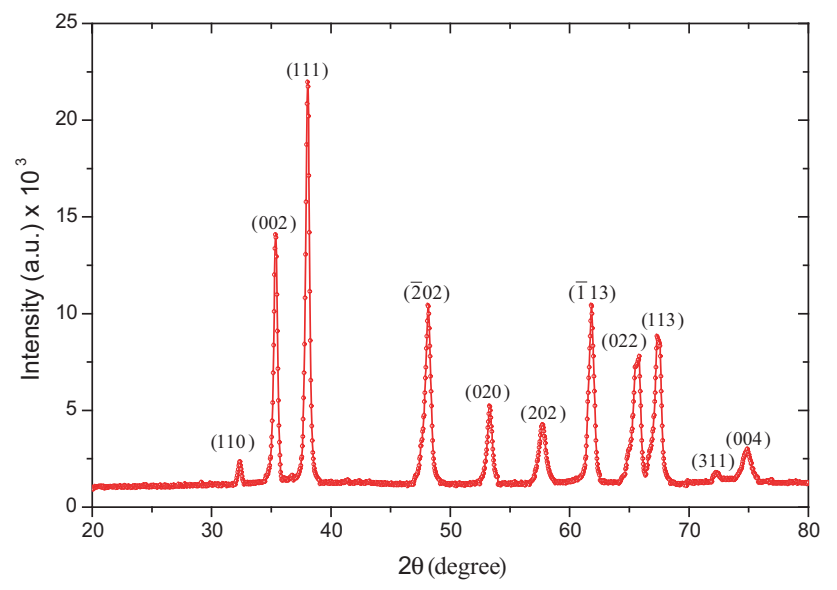

Figure 4. X-ray diffraction pattern of the sol-gel synthesized $\mathrm{CuO}$ nanoparticles.

On contrary, the intensity of characteristic peaks of DPE-WMR gradually increases. By comparing appropriate regions of figures 4 and 5 , it is found that the illustrated peaks in the range of $2 \theta=5-35^{\circ}$ are associated to DPE-W-MR, which are not detected in figure 3 .

The average particle size of DPE-W-MR/CuO di-layer thin films were calculated by the use of Debye-Scherrer's equation. The average crystallite sizes of DPE-W-MR/CuO di-thin films are 52.92, 56.24 and $72.26 \mathrm{~nm}$ for $T_{1}, T_{2}$ and $T_{3}$, respectively.

\subsection{SEM of the $\mathrm{CuO}$ thin film and DPE-W-MR/CuO di-layer thin film}

Figure 6a and $\mathrm{b}$ showed SEM image of $\mathrm{CuO}$ thin film with two different magnifications. The average particle size of copper

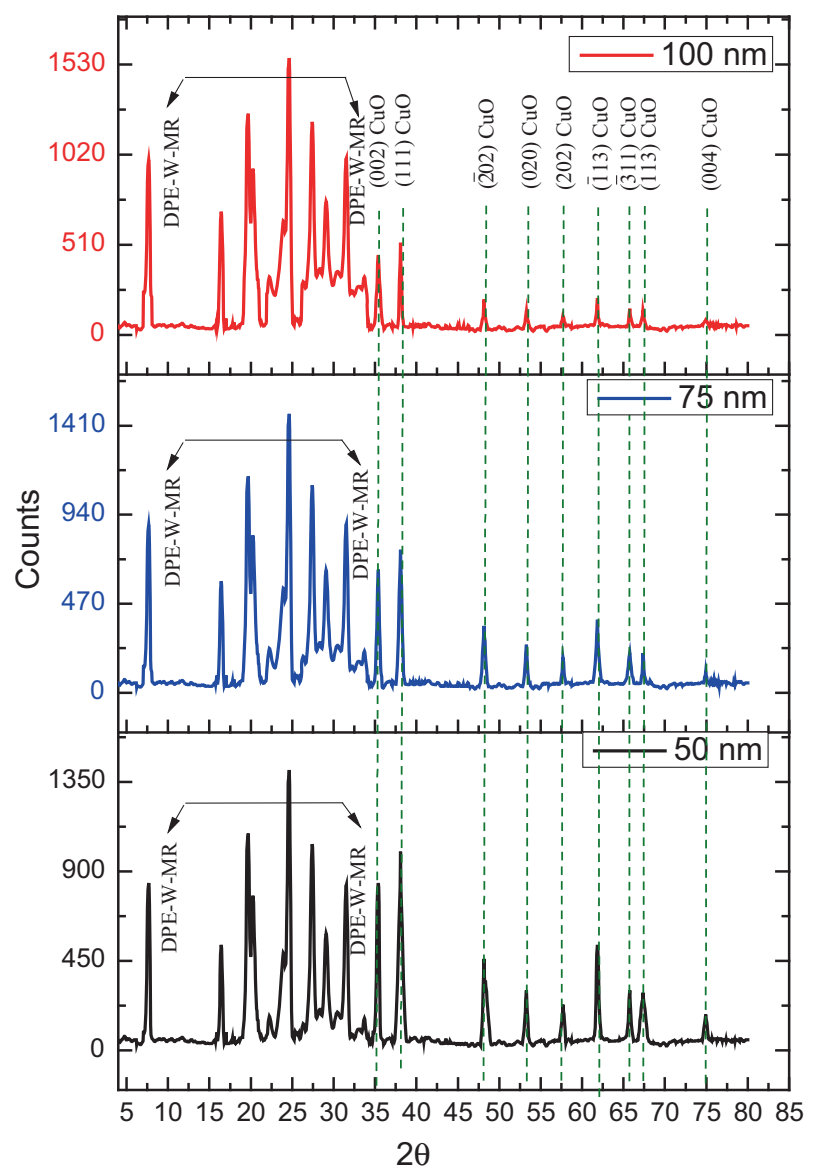

Figure 5. XRD diffraction pattern of the as-deposited of DPE$\mathrm{W}-\mathrm{MR} / \mathrm{CuO}$ di-layer thin films of $\mathrm{T}_{1}, \mathrm{~T}_{2}$ and $\mathrm{T}_{3}$. The intensity of the characteristic peaks of $\mathrm{CuO}$ gradually decreases from $\mathrm{T}_{1}$ to $\mathrm{T}_{2}$, whereas the intensity of characteristic peaks of DPE-W-MR gradually increases.

Table 2. The XRD data of copper oxide nanoparticles and DPE-W-MR/CuO di-layer thin films; miller indices ( $h k l)$, intensity, $d$-spacing, $\beta$-values and crystallite size, $D(\mathrm{~nm})$ as a function of $2 \theta$ position.

\begin{tabular}{|c|c|c|c|c|c|c|c|c|c|c|c|}
\hline \multirow[b]{2}{*}{$2 \theta$} & \multirow[b]{2}{*}{$h k l$} & \multirow[b]{2}{*}{ Intensity $\mathrm{CuO}$} & \multirow[b]{2}{*}{$d$-spacing $(\AA) \mathrm{CuO}$} & \multicolumn{4}{|c|}{$\beta(\mathrm{nm})$} & \multicolumn{4}{|c|}{$D(\mathrm{~nm})$} \\
\hline & & & & $\mathrm{CuO}$ & $50 / 50^{a}$ & $50 / 75^{b}$ & $50 / 100^{c}$ & $\mathrm{CuO}$ & $(50 / 50)^{a}$ & $(50 / 75)^{b}$ & $(50 / 100)^{c}$ \\
\hline 32.40 & (110) & 2344 & 2.760 & 0.13 & 0.12 & 0.12 & 0.11 & 63.69 & 69.00 & 69.00 & 75.27 \\
\hline 35.36 & (002) & 14082 & 2.535 & 0.28 & 0.26 & 0.24 & 0.23 & 29.80 & 32.10 & 34.77 & 36.28 \\
\hline 38.04 & (111) & 21968 & 2.363 & 0.33 & 0.31 & 0.28 & 0.26 & 25.48 & 27.13 & 30.04 & 32.35 \\
\hline 48.12 & $(\overline{2} 02)$ & 10430 & 1.889 & 0.36 & 0.33 & 0.31 & 0.25 & 24.19 & 26.39 & 28.09 & 34.83 \\
\hline 53.28 & $(020)$ & 5215 & 1.717 & 0.15 & 0.14 & 0.12 & 0.09 & 59.30 & 63.54 & 74.13 & 98.84 \\
\hline 57.72 & (202) & 4254 & 1.595 & 0.16 & 0.15 & 0.13 & 0.11 & 56.74 & 60.52 & 69.83 & 82.53 \\
\hline 61.8 & (1113) & 10430 & 1.499 & 0.22 & 0.2 & 0.19 & 0.15 & 42.12 & 46.33 & 48.77 & 61.77 \\
\hline 65.76 & $(022)$ & 7598 & 1.418 & 0.25 & 0.23 & 0.21 & 0.17 & 37.87 & 41.16 & 45.08 & 55.69 \\
\hline 67.32 & (113) & 8822 & 1.389 & 0.28 & 0.24 & 0.22 & 0.18 & 34.12 & 39.80 & 43.42 & 53.07 \\
\hline 72.24 & (311) & 1754 & 1.306 & 0.10 & 0.09 & 0.10 & 0.06 & 98.43 & 109.36 & 98.43 & 164.05 \\
\hline 74.92 & (004) & 2981 & 1.266 & 0.19 & 0.15 & 0.13 & 0.10 & 52.72 & 66.78 & 77.05 & 100.16 \\
\hline
\end{tabular}

${ }^{a}$ DPE-W-MR $(50 \mathrm{~nm}) /(50 \mathrm{~nm}) \mathrm{CuO}$.

${ }^{b}$ DPE-W-MR $(75 \mathrm{~nm}) /(50 \mathrm{~nm}) \mathrm{CuO}$.

${ }^{c}$ DPE-W-MR $(100 \mathrm{~nm}) /(50 \mathrm{~nm}) \mathrm{CuO}$ di-layer. 

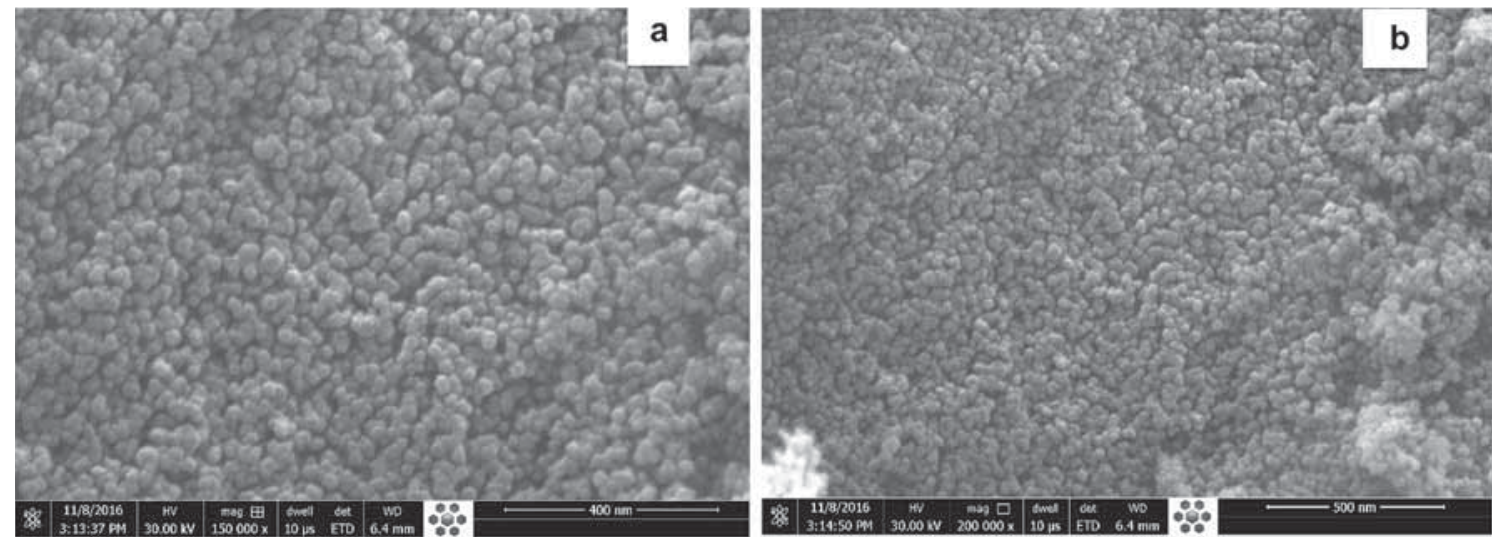

Figure 6. SEM of $\mathrm{CuO}$ nanostructure thin film $(50 \mathrm{~nm})$. SEM of $\mathrm{CuO}$ nanoparticles at scale bar of (a) 500 and (b) $400 \mathrm{~nm}$.
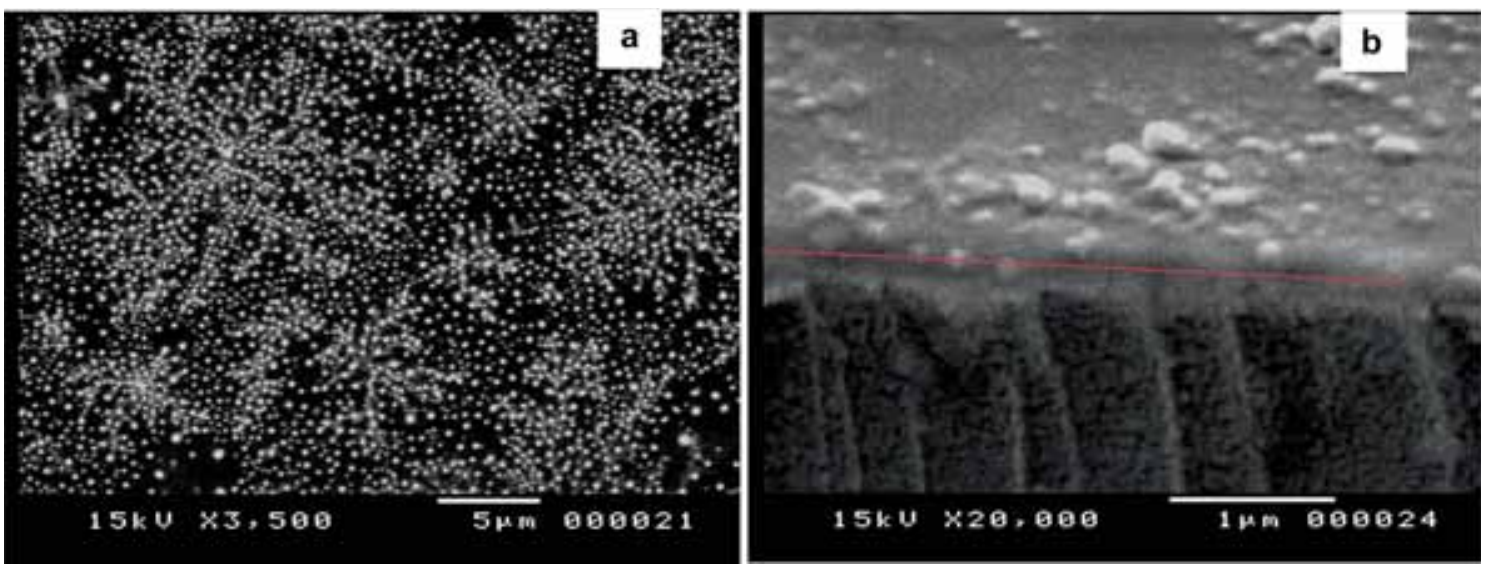

Figure 7. Selected SEM images of (a) DPE-W-MR di-layer thin film/CuO $\left(\mathrm{T}_{3}\right)($ scale bar $=5 \mu \mathrm{m})$ and (b) cross-section of $\left(\mathrm{T}_{3}\right)($ scale bar $=1 \mu \mathrm{m})$.

oxide thin film $\left(D_{\text {av }}\right)$ was found to be $\cong 53.12 \mathrm{~nm}$ with balllike shapes. The average particle size of copper oxide is in a good agreement with previous XRD data (table 2). Similar results were reported for ball-like $\mathrm{CuO}$ nanostructures synthesized by a hydrothermal method via controlling $\mathrm{pH}$ values of the solution [33].

Figure 7a shows the SEM micrographs of the surface asdeposited DPE-W-MR di-layer thin film/CuO $\left(\mathrm{T}_{3}\right)$. The SEM image shows that the thin film is flower-like and luminous with a homogeneous particle-size distribution. Cross-sectional dilayer thin film of $T_{3}$ was imaged by SEM (figure $7 b$ ) at $1 \mu \mathrm{m}$ scale bar. It can be observed that $\mathrm{CuO}$ layer is located below the red line, while DPE-W-MR layer is located on the upper surface. There are two different types of particle sizes, which can be ascribed to MR group and other diphenyl phosphine (DPE) group around tungsten atoms. In figure $7 \mathrm{a}$ and $\mathrm{b}$, the smoothness of the surface of the upper layer (DPE-W-MR) and its homogenous structure are clearly visible. Finally, the average nanoparticle size of DPE-W-MR di-layer thin film/ $\mathrm{CuO}\left(\mathrm{T}_{3}\right)$ determined by SEM images was found to be $96.45 \mathrm{~nm}$ in comparison with $72.26 \mathrm{~nm}$ (XRD data).

\subsection{Optical absorption of DPE-W-MR/CuO di-layer nanostructure thin films}

The absorbance and transmittance of the DPE-W-MR/CuO di-layer nanostructure films were recorded in the wavelength range of 250-2500 $\mathrm{nm}$ at room temperature $(\mathrm{K})$. The absorption spectra of the as-deposited DPE-W-MR/CuO di-layer films are depicted in figure 8. UV-visible spectra consist of two distinct regions: in the near ultraviolet (Soret bands) and visible regions ( $\mathrm{Q}$ bands). The multi-peaks of DPE$\mathrm{W}-\mathrm{MR} / \mathrm{CuO}$ di-layer films in the absorption spectra can be attributed to the vibrational excitation form of a ground state to an excited one. The absorption bands in DPE-W-MR/CuO di-layer films arise from transitions between two HOMOs and two LUMOs and it is the identities of the metal centre and the substituents on the ring that might affect the relative energies of these transitions. It is observed that the wavelength of 


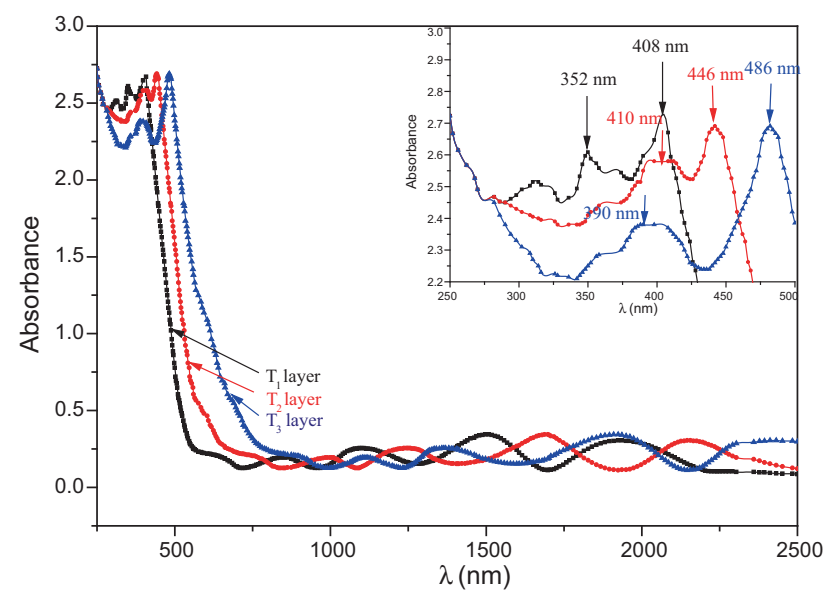

Figure 8. Optical absorption spectra of DPE-W-MR/CuO di-layer nanostructure thin films $\left(\mathrm{T}_{1}, \mathrm{~T}_{2}\right.$ and $\left.\mathrm{T}_{3}\right)$. Inset is $\mathrm{UV}$ wavelength region at a wavelength of 250-500 $\mathrm{nm}$ for DPE-W-MR/CuO di-layer nanostructure films.

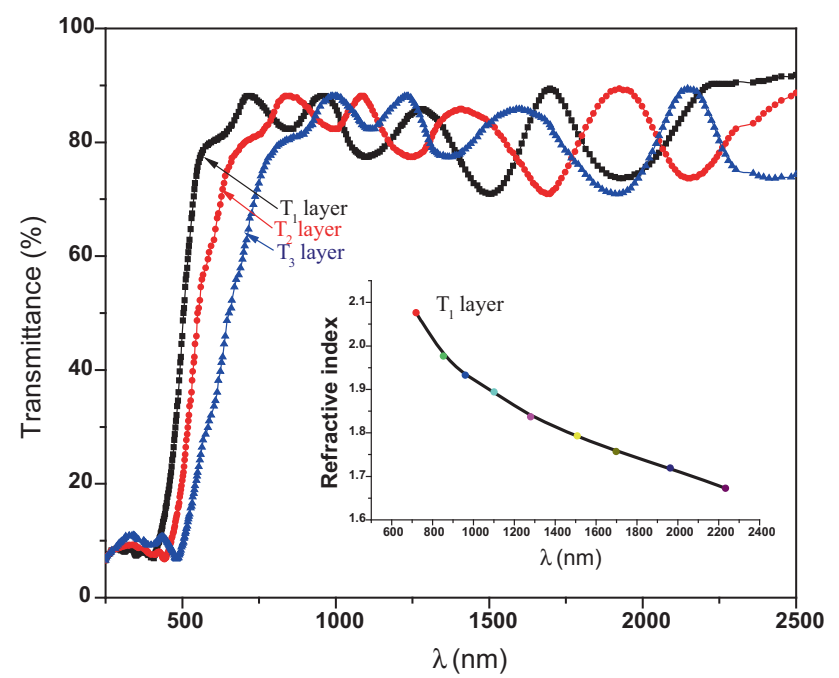

Figure 9. Optical transmission spectra of as-deposited DPE-W$\mathrm{MR} / \mathrm{CuO}$ di-layer thin film; $\mathrm{T}_{1}$ : black, $\mathrm{T}_{2}$ : red and $\mathrm{T}_{3}$ : blue. Inset is the dependence of the complex refractive index $(n)$ on the wavelength $(\lambda, \mathrm{nm})$.

the as-deposited DPE-W-MR/CuO di-layer films varies from 250 to $500 \mathrm{~nm}$ from $\mathrm{T}_{1}$ to $\mathrm{T}_{3}$ (figure 8 inset). The absorption peaks (two weak peaks) appear in the UV wavelength region at wavelength of $250-500 \mathrm{~nm}$ for DPE-W-MR/CuO di-layer nanostructure films.

From figure 9, it can be noticed that the absorption peaks shift towards longer wavelength as the DPE-W-MR layer thickness increases from $T_{1}$ to $T_{3}$. The particle size and the quantum confinement effects of DPE-W-MR layers have the major effect to the red shift in the absorption edge of the semiconductors. Therefore, one-dimensional confinement in the layers was also responsible for enhanced quantum confinement effects with the increase in DPE-W-MR layer thickness (except for the formation of nanostructure film).

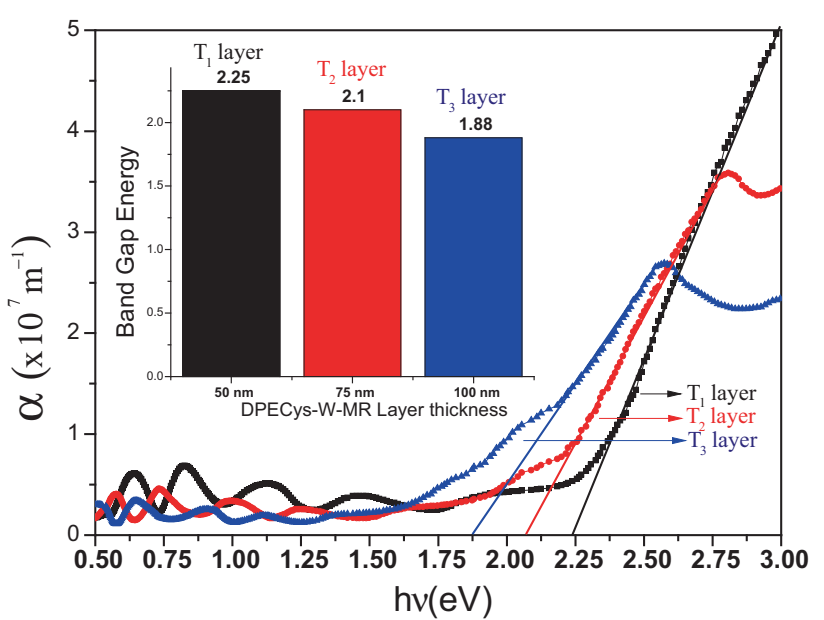

Figure 10. Plots of absorption coefficient, $\alpha$, as a function of photon energy, $h v$, of as-deposited di-layer thin film $\left(\mathrm{T}_{1}, \mathrm{~T}_{2}\right.$ and $\left.\mathrm{T}_{3}\right)$. Inset: the band gap of DPE-W-MR/CuO di-layer thin films were $2.25,2.1$ and $1.88 \mathrm{eV}$ for $T_{1}, T_{2}$ and $T_{3}$, respectively.

Experimentally, the absorption coefficient $(\alpha)\left(\mathrm{m}^{-1}\right)$ was calculated by the following relationship [34]

$$
\alpha=\frac{1}{d} \ln \left(\frac{1}{T}\right)
$$

where $d$ is the thickness of the film (in $\mathrm{cm}$ ) and $T$ is the transmitted intensity of the film layer. Figure 10 shows the plot of absorption coefficient $(\alpha)$ (lies in between 0 and 5 $\left(\times 10^{7} \mathrm{~m}^{-1}\right)$ ) with photon energy $(h v)$. Hence, the band gap of DPE-W-MR/CuO di-layer thin films was 2.25, 2.1 and 1.88 $\mathrm{eV}$ for $\mathrm{T}_{1}, \mathrm{~T}_{2}$ and $\mathrm{T}_{3}$, respectively (figure $10 \mathrm{inset}$ ).

The optical properties of $T_{1}$ film were evaluated from transmittance data using Myburg [35] method. Swanepoel's [36] method is employed for spectroscopic determination of optical properties of DPE-W-MR/CuO di-layer thin films using transmittance data do not have 'Swanepoel' author name. The application of the above method is used to calculate the maximum and minimum transmittance envelope curves by parabolic interpolation to the experimentally determined positions of peaks and valleys. Figure 9 shows that the values of refractive index $(n)$ decrease with an increase in wavelength, $\lambda(\mathrm{nm})$. The real values of refractive index $\left(n_{\mathrm{s}}\right)$ depended on maximum and minimum transmittance points are calculated using the expression proposed by Swanepoel's [35] as given below:

$$
n=\left[N+\sqrt{N^{2}-n_{\mathrm{s}}^{2}}\right]^{1 / 2}
$$

In the weak and medium absorption regions, the value of $N$ is given by the following equation:

$$
N=2 n_{\mathrm{s}} \frac{T_{\max }-T_{\min }}{T_{\max } T_{\min }}+\frac{n_{\mathrm{s}}^{2}+1}{2} .
$$


Table 3. Values of wavelength, $\lambda(\mathrm{nm}), T_{\max }$ and $T_{\min }$ for the transmittance spectrum of $\mathrm{T}_{1}$ of as-deposited multi-layer DPE-W-MR/CuO di-layer films and refractive index $(n)$ to the equivalent wavelength, $\lambda(\mathrm{nm})$.

\begin{tabular}{llll}
\hline$\lambda(\mathrm{nm})$ & $T_{\max }$ & \multicolumn{1}{c}{$T_{\min }$} & \multicolumn{1}{c}{$N$} \\
\hline 720 & 0.81133 & 0.780401 & 2.076383 \\
854 & 0.83803 & 0.80374 & 1.976471 \\
960 & 0.84133 & 0.81506 & 1.932849 \\
1102 & 0.85209 & 0.82447 & 1.893732 \\
1280 & 0.86833 & 0.83815 & 1.836922 \\
1508 & 0.87822 & 0.84913 & 1.792917 \\
1698 & 0.89437 & 0.85689 & 1.756996 \\
1963 & 0.901034 & 0.86674 & 1.718866 \\
2234 & 0.91229 & 0.87821 & 1.672258 \\
\hline
\end{tabular}

The $n_{\mathrm{s}}$ value being the refractive index of the quartz substrate. Generally, the $n_{\mathrm{s}}$ value is calculated by the maximum of the transmission in the transparent region $T_{\max }$ using the equation [37]:

$$
n_{\mathrm{s}}=\frac{1}{T_{\max }}+\sqrt{\left(\frac{1}{T_{\max }^{2}}-1\right)} .
$$

The refractive index $(n)$ values at the extremes of the spectra of wavelength $(\lambda, \mathrm{nm}), T_{\max }$ and $T_{\min }$ are obtained from the plot of figure 9 . The applied optical method, the dependence of the complex refractive index $(n)$ on the wavelength $(\lambda)$ is shown in figure 9 (inset). A sharp fall in the refractive index $(n)$ for $T_{1}$ is observed at the lower wavelength region and a gradual destruction is observed for the refractive index corresponding to the higher wavelength region. The values of refractive index $(n)$ calculated from equation (2) are resented in table 3 . This variation in refractive index indicates a normal dispersive behaviour of $T_{1}$ thick as-deposited multi-layer DPE-W-MR/CuO di-layer nanostructure films [38-41].

\section{Conclusions}

Nanostructured DPEs-W-MR/CuO di-layer thin films were prepared by sol-gel spin-coating technique. Three sets of DPE-W-MR/CuO di-layer films were deposited on quartz substrates by spin-coating process with different DPE-WMR layer thicknesses (50, 75 and $100 \mathrm{~nm}$ ) and fixed $\mathrm{CuO}$ layer thickness $(50 \mathrm{~nm})$. The composition and the chemical structure of the fabricated thin films were characterized using a combination of spectroscopic and diffraction techniques. The particle sizes of $\mathrm{CuO}$ nanoparticles and thin films were calculated by applying Debye-Scherrer's formula. The optical band gap values for thin films varied from 1.88 to $2.25 \mathrm{eV}$ depending upon the layer's thickness of thin films.
The variations of the refractive index $(n)$ with the wavelengths was determined. SEM observations of DPE-W-MR/CuO di-layer thin films revealed that agglomeration of the Wphosphine/ $\mathrm{CuO}$ clusters to form spherical $\mathrm{CuO}$ nanoparticles. The thermal characteristics (TGA) of DPE-W-MR complexes were discussed. The XRD data and optical characteristics of DPE-W-MR thin films show strong dependence on the film thickness. The structural and optical characteristics of DPE-W-MR thin films are of high relevance, especially for potential applications in semiconductor optoelectronic device development

\section{Acknowledgement}

This study was funded by Faculty of Science, New Valley Branch, Assiut University (AUN) and Northern Border University (NBU), Arar. We, therefore, acknowledge with thanks AUN and NBU financial support.

\section{References}

[1] Liu Z, Liu G, Liu X, Huang S, Liu M, Fu Guolan et al 2015 Mater. Lett. 13912

[2] Hosny N M, Samir G, Zoromba M S and Alghool S $2017 \mathrm{~J}$. Polym. Plast. Technnol. Eng. 56435

[3] Zoromba M S, Bassyouni M and Abdel-Hamid S 2015 Rubber Chem. Technol. 88449

[4] Zoromba M S, Ismail M I M, Bassyouni M, Abdel-Aziz M H, Numan S, Alshahrie A et al 2017 Coll. Surf. A Phys. Chem. Eng. Asp. $\mathbf{5 2 0} 121$

[5] Al-Hossainy A F 2016 Bull. Mater. Sci. 39209

[6] Pate J, Zamora F, Watson M D S, Wright G N, Horrocks B and Houlton A 2014 J. Mater. Chem. C 29265

[7] Liao Y-C and Kao Z-K 2012 ACS Appl. Mater. Interface 10 5109

[8] Liu T, Vilar R, Eugénio S, Joseph G and Yann D 2015 J. Appl. Electrochem. 4587

[9] Yang-Kai H, Shih-Pin C, Su C-H and Liao Y-C 2015 Sci. Adv. Mater. 7227

[10] Volpati D, Spada E R, Plá Cid C C, Sartorelli M L, Aroca R and Constantino C 2015 Analyst 140476

[11] Yonezawa Y, Takami A and Sato T 1990 J. Appl. Phys. 681297

[12] Griffiths M, Pallister P, Mandia D and Barry S 2015 Chem. Mater. 2844

[13] Hyeong-Chul Y, Jin-Ha S, Park H-S and Su-Jeong S $2015 \mathrm{~J}$. Nanosci. Nanotechnol. 151601

[14] Nagai H, Mita S, Takano I, Honda T and Sato M 2015 Mater. Lett. 141235

[15] Ghotbi M and Rahmati Z 2015 Mater. Des. 85719

[16] Walker S D, Barder T E, Martinelli J and Buchwald S 2004 Angew. Chem. Int. Ed. 431871

[17] Caminade A-M, Maraval V, Laurent R and Majoral J-P 2002 Curr. Org. Chem. 6739

[18] Iwasawa T, Tokunaga M, Obora Y and Tsuji Y 2004 J. Am. Chem. Soc. 1266554 
[19] Niyomura O, Iwasawa T, Sawada N, Tokunaga M, Obora Y and Tsuji Y 2005 Organometallics 243468

[20] Chen J, Law C, Lam J, Dong Y, Lo S, Williams I et al 2003 Chem. Mater. 151535

[21] Verma A, Hirsch D, Glatt C, Ronnett G and Snyder S 1993 Sci. Wash. 259381

[22] Clark J, Naughton P, Shurey S, Green C, Johnson T, Mann B et al 2003 Circ. Res. 93 e2

[23] Awad I, Hassan F, Mohamed A and Al-Hossainy A $2004 \mathrm{~J}$. Phosphorus Sulfur Silicon 1791251

[24] Hassan F, Al-Hossainy A and Mohamed A 2009 J. Phosphorus Sulfur Silicon 1842996

[25] Badr M, El-Amin A and Al-Hossainy A 2008 J. Phys. Chem. C 11214188

[26] Badr M, El-Amin A and Al-Hossainy A 2006 Eur. Phys. J. B 53 439

[27] Takakazu Y, Yoshio E, Minoru K and Akio Y 1980 Bull. Chem. Soc. Jpn. $\mathbf{5 3} 1299$

[28] Maury R, Jhamb S, Roy S, Chourasia J, Sharma A and Vishwakarma P 2015 Arab. J. Chem. 8143

[29] El-Morsy F, Jean-Claude B, Butler I, El-Sayed S and Mostafa S 2014 Inorg. Chim. Acta $\mathbf{4 2 3} 144$
[30] El-Hendawy A M, El-Kourashy A E-G and Shanab M M 1992 Polyhedron 11523

[31] Santra P, Sinha C, Sheen W-J, Liao F-L and Lu T-H 2001 Polyhedron 20599

[32] Maurya M, Jayaswal M, Puranik V, Chakrabarti P, Gopinathan S and Gopinathan 1997 Polyhedron 163977

[33] Chand P, Gaur A and Kumar A 2013 Superlattices Microstruc. 60129

[34] Valentini A, Nappi E and Nitti M 2002 Nucl. Instrum. Methods Phys. Res. A 482238

[35] Myburg G and R Swanepoel 1987 J. Non-Cryst. Solids 89 13

[36] Minkov D 1989 J. Phys. D Appl. Phys. 22199

[37] Manifacier J, Gasiot J and Fillard J 1976 J. Phys. E Sci. Instrum. 91002

[38] Zoromba M, Al-Hossainy A and Abdel-Aziz M 2017 Synth. Met. 23134

[39] Trilok, Rai R and Singh B K 2015 Nucl. Instrum. Methods Phys. Res. A 78570

[40] Al-Hossainy A and Ali I 2015 Opt. Mater. 46131

[41] Al-Hossainy A and Ali I 2015 Mater. Sci. Semicond. Proc. 38 13 